\title{
Effect of Non-Steroidal Anti-Inflammatory Drugs on Bone Healing and Osseointegration: the Need for large Scale Human Clinical Trials
}

\author{
Research Article
}

Volume 1 Issue 2- 2021

\begin{abstract}
Author Details
Colin Burns ${ }^{1}$, Siwei $\mathrm{Li}^{2}$, Fadi Barrak ${ }^{3}$

${ }^{1}$ Honorary Lecturer, School of Dentistry, University of Central Lancashire, Preston, United Kingdom PR1 2HE

${ }^{2}$ Visiting Academic, Imperial College London, London SW7 2AZ; Research Project Manager, Visiting Specialist Services

Ltd, 27 Old Gloucester Street, London, WC1N 3AX

${ }^{3}$ Director, Visiting Specialist Services Ltd, 27 Old Gloucester Street, London, WC1N 3AX; Clinical Lead, School of

Dentistry, University of Central Lancashire, Preston, United Kingdom PR1 2HE

*Corresponding author
\end{abstract}

Fadi Barrak, Director, Visiting Specialist Services Ltd, 27 Old Gloucester Street, London, WC1N 3AX; Clinical Lead, School of Dentistry, University of Central Lancashire, Preston, United Kingdom PR1 2HE

Article History

Received: August 23, 2021 Accepted: September 01, 2021 Published: September 02, 2021

\begin{abstract}
This article revisits the topic whether the use of non-steroidal anti-inflammatory drugs (NSAIDs) affect bone healing and osseointegration. An understanding on this topic is crucial for clinicians to make evidence-based decisions to ensure patient safety and long-term success of implants. Based on authors' systematic search, a limited number of articles were found to merit another systematic review. The understanding on the effects of NSAIDs on bone, specifically in human subjects, and the underlying biochemical mechanism, remain limited, owing to design variations in limited published studies. Some studies may suggest NSAIDs have no adverse, if not protective effects. One can suggest that a combination of certain NSAID type, dosage, administration timing and duration may adversely affect bone. Authors would like to raise awareness and highlight the need of collective efforts and further studies with standardised quantitative measurements to help our understanding of the effects of this commonly used line of treatment.
\end{abstract}

Keywords: Non-steroidal anti-inflammatory drugs; NSAIDs; Implant; Hypoxia; Cyclooxygenase inhibitor; Osseointegration

\begin{abstract}
Abbreviations: NSAIDs: Non-Steroidal Anti- Inflammatory Drugs; PG: Prostaglandin; COX: Cyclooxygenase; RANKL: Receptor Activator of Nuclear Factor- $\kappa B$; NFATc1: Nuclear Factor of Activated T Cell Cytoplasmic 1; NF- $\mathrm{B}$ : Nuclear Factor Kappa B; HIF: HypoxiaInducible Factor; BMP2: Bone Morphogenetic Protein 2
\end{abstract}

\section{Introduction}

The use of dental implants has become an integral part for the treatment of partial and complete edentulism [1]. Single stage protocol, where the implant is immediately loaded following its insertion and the installation of prosthetic tooth, is gaining popularity due to its potential in reduction of number of surgical interventions required while providing favorable clinical outcomes [2]. Optimal peri-implant bone healing during the early phases of osseointegration is crucial in providing stability and hence the long-term reliability of the treatment [3]. However, peri-implant bone healing can be affected by the quantity and quality of the bone, which is known to be affected by the patient's systemic health and certain medical conditions [4].

Nonsteroidal anti-inflammatory drugs (NSAIDs) are regularly used globally to control chronic pain and/or inflammatory burden. In addition, both over the counter and prescription NSAIDs are extensively used as post-operative analgesics in the U.K. The effects of NSAIDs on bone remodelling and/or healing, however, are often overlooked when used as post-operative pain management. Prostaglandins (PGs) synthesised from arachidonic acid (from phospholipids bilayer in cell membrane) via cyclooxygenase (COX) activity mediate the destruction and formation of bone in the remodelling process [5]. Multiple COX isoforms (COX-1 and COX-2) exist, albeit the exact reason is an unanswered question. Depending on the type, NSAIDs can either selectively or non-selectively inhibit the activity of COX-1 and/or COX-2 and thereby influence the bone healing cascade [6]. 
Although there is evidence from in vitro and animal studies that indicate COX inhibitors, particularly, COX-2 inhibitors adversely affect bone formation [7-9], the exact role of COX enzymes and the effect of their inhibition in humans has not been ascertained. Several systematic reviews on the effect of NSAIDs on osseointegration in both orthopaedic and dental fields have been published [1013], however, to date no consensus on this subject can be reached. The lack of information on co-factors such as smoking, alcohol consumption and other underlying conditions in many published studies exacerbate uncertainties. Through authors' own systematic literature search, several articles, which were not discussed in current published systematic reviews, have been identified. These limited findings, however, do not merit another systematic review. Therefore, authors would like to express our views and raise awareness through this opinion paper that the effect of NSAIDS on osseointegration is dependent on multiple factors and hence, carefully designed further research is needed.

\section{Methods and Materials}

\section{Literature search}

Following the initial scoping searches and formulation of the research question, in May 2020 a PRISMA (preferred reporting Items for systematic reviews and meta-analyses) workflow was used to search relevant publications in PubMed, Medline Ovid and Web of Knowledge search engines. The Search Terms used were: (antiinflammatory agents, non-steroidal[Pharmacological Action]) OR (anti-inflammatory agents, non-steroidal [MeSH Terms]) OR ((antiinflammatory) AND (agents) AND (non-steroidal)) OR (non-steroidal anti-inflammatory agents) OR (nsaid) OR (nsaids) OR (nsaid's) OR ((non) AND (steroidal) AND (anti) AND (inflammatory) AND (drug)) OR (non steroidalanti inflammatory drug) AND ((dental implants [MeSH Terms]) OR ((dental) AND (implants)) OR ((dental) AND (implant)) OR (dental implant) OR (dental implants)).

\section{Inclusion and exclusion criteria}

Inclusion Criteria included: osseointegration as primary/secondary outcome, randomised controlled trials, controlled clinical trials, human trials / animal trials and English language. Non-English literatures were excluded.

\section{Results}

Full-text articles of 17 studies were obtained and analysed. 9 articles were excluded as osseointegration was not defined as a primary or secondary outcome. The remaining 8 articles were assessed for this short communication.

\section{Discussion}

Two most commented human clinical trials in currently published systematic reviews are by Alissa et al. [14] and Sakka et al. [15], wherein non-selective NSAIDS ibuprofen (both $600 \mathrm{mg} 4$ times/day for 7 days post operation) were used. Neither study demonstrated statistically significant difference in bone levels in comparison to placebo or non-ibuprofen groups up to 6 months post operation. Authors further identified a human clinical trial by Bichara et al. [16], in which naproxen 500mg twice/day for 7 days was prescribed post operation and similarly, no significant difference in bone fill at defect site was reported up to 9 months post operation in naproxen and control groups. Interestingly, in a clinical trial by Jeffcoat et al. that used flubiprofen either $50 \mathrm{mg}$ or $100 \mathrm{mg}$ twice/day for 7 days post operation, it was reported that high dose flurbiprofen reduced $(\mathrm{p}<0.05)$ the amount of bone loss up to 12 months post operation in comparison to low dose or placebo group [17]. Similarly, authors identified yet another clinical trial by Reddy et al. [18], in which patients who received $100 \mathrm{mg}$ flubiprofen twice/day for 7 days resulted in increased bone density surrounding dental implants in comparison to control group, albeit the analyses were based on qualitative radiographs only. It is therefore not unreasonable to suspect that low dose use of flurbiprofen may result in adverse effects on bone.

Overall, there is insufficient evidence in the current literature to allow explicit conclusion on the effect of NSAIDs on osseointegration and/ or implant failure. This is due to the limited literature available and discrepancies owing to variables such as dosage, dosage timing, dosage duration, choice of NSAIDs family, test species, age, sex, measurement endpoints as well as primary outcome measures. However, it may be deceptive to postulate that NSAIDS, as a class of drugs, due to their ability to inhibit COX and PGs, have adverse effects on bone and indeed, the impedance of bone healing that has been reported frequently in animal models has not always been translated to human clinical trials. Based on available literature, one can infer that different types of NSAIDs exert different effects on bone modelling, therefore further understanding of biochemical mechanisms underlying these drugs are needed.

It has been reported that flurbiprofen and its derivatives can inhibit osteoclast activation and osteoclast mediated bone resorption, through a mechanism independent of COX $[19,20]$.This could explain, at least partially, the positive effects of flurbiprofen, when used at high dose, on bone that were seen in studies by Jeffcoat et al. [17] and Reddy et al. [18]. It should be noted that both studies prescribed flurbiprofen for a duration of 90 days, it is perhaps beneficial for patients who require long term use of NSAIDs for chronic pain relief to use flurbiprofen when an implant treatment is needed. Further studies are required to support such theory and investigate the effects of short term use of NSAIDS. It also appears that the positive effects of flurbiprofen is dose dependant [17]. Although the current literature is limited, it ispossible that other NSAIDs also have dose dependent effects on bone. For example, low dose aspirin $(<100 \mu \mathrm{g} / \mathrm{ml}$, equivalent to approximately $100 \mathrm{mg}$ in human), has been shown to be beneficial for maintaining bone mass through inhibition of receptor activator of nuclear factor- $\mathrm{\kappa B}$ ligand (RANKL) induced osteoclast activities via nuclear factor kappa $B(N F-\kappa B)$ and nuclear factor of activated $T$ cell cytoplasmic 1 (NFATc1) pathways that are again independent of COX $[21,22]$. It is, therefore, possible that low dose NSAIDs should be used in combination with other biochemical factors to achieve pain relief while minimising the risk of bone loss. In addition to COX-1 and COX2, the signalling pathway of hypoxia-inducible factor (HIF), in particular HIF-1区 subunit, is crucial in the regulation of osteoblast activity in bone repair [23]. While non-selective NSAID such as ibuprofen has been shown to reduce the expression of HIF in certain cell types [24,25], it has been reported that activation of HIF can attenuate the periapical inflammation, bone loss and stimulate heterotopic ossification [26,27]. Hypoxia-mimetic agents such as cobalt chloride can potentially be used in combination with NSAIDs to mitigate the possible negative effects on bone [28].

Further, while most of the animal and human studies focused on the post-operative administration of NSAIDs on bone healing, there are limited studies on the effects of pre-operative use of NSAIDs. One study by Lupepsa et al. [21] demonstrated that pre-operative use (42 days) of low dose $(6.75 \mathrm{mg} / \mathrm{kg}$ in rat, approximately equivalent of 75 $80 \mathrm{mg}$ in human) non-selective NSAID aspirin resulted in an impaired bone deposition during early stage (7 days) of bone repair after implant placement [21]. Interestingly, the adverse effects were not detected after 28 days. This may partially explain the clinical trials conducted by Alissa et al. [14], Jeffcoat et al. [17] and Sakka et al. [15] did not reveal adverse effect of ibuprofen on bone, as these studies only examined patients at one time point 3 to 6 months post operation. It is possible the effect of NSAIDs on post operation bone healing occur primarily during the early stage and, it is uncertain whether any change in earlystage bone healing has a long-term effects on bone biochemically and/ 
or mechanically. This again highlights the need of well-designed and long-term future animal studies as well as clinical trials.

Lastly, with the advent of selective COX-2 inhibitors and their nominal advantages including lower potential for causing gastrointestinal bleeding, more clinicians may use selective COX-2 inhibitors in favour of traditional non-selective NSAIDs [29,30]. Similar to nonselective NSAIDs, the effects of COX-2 inhibitors on bone healing and osseointegration remain highly debated, owing to discrepancies in aforementioned variables in study designs. However, it is worth noting that there is an increasing number of reports that suggest the effects selective COX-2 inhibitors are detrimental to bone or facture healing [31-33]. It has been suggested that selective COX-2 inhibitors such as celecoxib inhibit bone morphogenetic protein 2 (BMP2) regulated osteoblast differentiation independent of COX activity [34], therefore, caution should be used when prescribing selective COX-2 inhibitors.

\section{Conclusion}

The limitations of our understanding on the effects of NSAIDs on bone, specifically in human subjects, are due to the wide variations in the designs of limited number of published studies. The exact effect of NSAIDs, as a class of drugs, on bone remain debatable. Although some available evidence may suggest NSAIDs have no adverse, if not protective effects on bone, one can suggest that a combination of certain type of NSAID drug, dosage, administration timing as well as duration may adversely affect bone. In addition, the question whether NSAIDs affect bone remain particularly relevant for patients on long term NSAIDs and possibly, will also be dependent on loading protocol following implant insertion. A collective effort and further studies with direct comparison of NSAIDs and control groups in well-designed studies with standardised quantitative measurement techniques are needed to help our understanding of the effects of this commonly used line of treatment.

\section{Acknowledgements}

Not applicable

\section{Competing Interests}

The authors declare that they have no competing interests.

\section{Funding}

Not applicable.

\section{References}

1. Block MS (2018) Dental Implants: The Last 100 Years. J Oral Maxillofac Surg 76(1): 11-26.

2. Del Fabbro M, Testori T, Kekovic V (2019) A Systematic Review of Survival Rates of Osseointegrated Implants in Fully and Partially Edentulous Patients Following Immediate Loading. J Clin Med 8(12).

3. Lioubavina-Hack N, Lang NP, Karring T (2006) Significance of primary stability for osseointegration of dental implants. Clin Oral Implants Res17(3): 244-250

4. Fu JH, Bashutski JD, Al-Hezaimi K (2012) Statins, glucocorticoids, and nonsteroidal anti-inflammatory drugs: their influence on implant healing. Implant Dent 21(5): 362-367.

5. Blackwell KA, Raisz LG, Pilbeam CC (2010) Prostaglandins in bone: bad cop, good cop? Trends Endocrinol Metab 21(5): 294-301.

6. Gupta PJ (2007) Effects of warm water sitz bath on symptoms in postanal sphincterotomy in chronic anal fissure--a randomized and controlled study. World J Surg 31(7): 1480-1484.

7. Muller M, Raabe O, Addicks K (2011) Effects of non-steroidal antiinflammatory drugs on proliferation, differentiation and migration in equine mesenchymal stem cells. Cell Biol Int 35(3): 235-248.
8. Park J, Fertala A, Tomlinson RE (2019) Naproxen impairs load-induced bone formation, reduces bone toughness, and diminishes woven bone formation following stress fracture in mice. Bone 124: 22-32.

9. Damrongsri D, Geva S, Salvi GE (2006) Cyclooxygenase-2 inhibition selectively attenuates bone morphogenetic protein- 6 synthesis and bone formation during guided tissue regeneration in a rat model. Clin Oral Implants Res 17(1): 38-47

10. Marquez-Lara A, Hutchinson ID, Nunez F Jr (2016) Nonsteroidal AntiInflammatory Drugs and Bone-Healing: A Systematic Review of Research Quality. JBJS Rev 4(3).

11. Dave M, Patel N (2020) A systematic review to determine the impact of non-steroidal anti-inflammatory drugs on dental implant osseointegration. Oral Surgery 13(1): 57-66.

12. Luo JD, Miller C, Jirjis T (2018) The effect of non-steroidal antiinflammatory drugs on the osteogenic activity in osseointegration: a systematic review. Int J Implant Dent 4(1): 30.

13. Alhindi M, Muaddi H, Almosairy M (2018) The Influence of NSAIDS on Osseointegration of Dental Implants A Systematic Review. Biomed J Sci \& Tech Res.

14. Alissa R, Sakka S, Oliver R (2009) Influence of ibuprofen on bone healing around dental implants: a randomised double-blind placebo-controlled clinical study. Eur J Oral Implantol 2(3): 185-199.

15. Sakka S, Hanouneh SI (2013) Investigation of the effect of ibuprofen on the healing of osseointegrated oral implants. J Investig Clin Dent 4(2): 113-119.

16. Bichara J, Greenwell H, Drisko C (1999) The effect of postsurgical naproxen and a bioabsorbable membrane on osseous healing in intrabony defects. J Periodontol 70(8): 869-877.

17. Jeffcoat MK, Reddy MS, Wang IC (1995) The effect of systemic flurbiprofen on bone supporting dental implants. J Am Dent Assoc 126(3): 305-311.

18. Reddy MS, Jeffcoat MK, Richardson RC (1990) Assessment of adjunctive flurbiprofen therapy in root-form implant healing with digital subtraction radiography. J Oral Implantol 16(4): 272-276.

19. Idris AI, Del Soldato P, Ralston SH (2004) The flurbiprofen derivatives HCT1026 and HCT1027 inhibit bone resorption by a mechanism independent of COX inhibition and nitric oxide production. Bone 35(3): 636-643.

20. Anai K, Tatsumi J, Kurihara H (1993) Flurbiprofen Inhibits Osteoclast Formation and Osteoclast Mediated Bone Resorption. Journal of the Japanese Society of Periodontology 35(4): 655-660.

21. Lupepsa AC, Vargas-Sanchez P, Moro MG (2021) Effects of low-dose aspirin on the osseointegration process in rats. Int J Implant Dent 7(1): 3 .

22. Wu L, Luo Z, Liu Y (2019) Aspirin inhibits RANKL-induced osteoclast differentiation in dendritic cells by suppressing NF-kappaB and NFATcl activation. Stem Cell Res Thern 10(1): 375 .

23. Lisowska B, Kosson D, Domaracka K (2018) Lights and shadows of NSAIDs in bone healing: the role of prostaglandins in bone metabolism. Drug Des Devel Ther 12: 1753-1758.

24. Sun Y, Cai N, Liu N (2017) Celecoxib Down-Regulates the HypoxiaInduced Expression of HIF-1 $\alpha$ and VEGF Through the PI3K/AKT Pathway in Retinal Pigment Epithelial Cells. Cellular Physiology and Biochemistry 44(4): 1640-1650.

25. Palayoor ST, Tofilon PJ, Coleman CN (2003) Ibuprofen-mediated reduction of hypoxia-inducible factors HIF-1alpha and HIF-2alpha in prostate cancer cells. Clin Cancer Res 9(8): 3150-3157.

26. Hirai K, Furusho H, Hirota K (2018) Activation of hypoxia-inducible factor 1 attenuates periapical inflammation and bone loss. International Journal of Oral Science 10(2): 12.

27. Huang $\mathrm{Y}$, Wang $\mathrm{X}$, Lin $\mathrm{H}$ (2020) The hypoxic microenvironment: a driving force for heterotopic ossification progression. Cell Commun Signal 18(1): 20 . 
28. Teti G, Focaroli S, Salvatore V (2018) The Hypoxia-Mimetic Agent Cobalt Chloride Differently Affects Human Mesenchymal Stem Cells in Their Chondrogenic Potential. Stem Cells International 18: 3237253.

29. Rahme E, Nedjar H (2007) Risks and benefits of COX-2 inhibitors vs non-selective NSAIDs: does their cardiovascular risk exceed their gastrointestinal benefit? A retrospective cohort study. Rheumatology 46(3): 435-438.

30. Rana MV, Desai R, Tran L (2016) Perioperative Pain Control in the Ambulatory Setting. Curr Pain Headache Rep 20(3): 18.

31. Cottrell J, O'Connor JP (2010) Effect of Non-Steroidal Anti-Inflammatory Drugs on Bone Healing. Pharmaceuticals (Basel) 3(5): 1668-1693.
32. Jiang M, Deng H, Chen X (2020) The efficacy and safety of selective COX2 inhibitors for postoperative pain management in patients after total knee/hip arthroplasty: a meta-analysis. J Orthop Surg Res 15(1): 39.

33. Etikala A, Tattan M, Askar H (2019) Effects of NSAIDs on Periodontal and Dental Implant Therapy. Compend Contin Educ Dent 40(2): e1-e9.

34. Matsuyama A, Higashi S, Tanizaki S (2018) Celecoxib inhibits osteoblast differentiation independent of cyclooxygenase activity. Clin Exp Pharmacol Physiol 45(1): 75-83 